\title{
VAC_14 - Immunogenicity of a 17-DD Yellow Fever Vaccine in a dengue and Zika endemic area of Paraiba State, Brazil
}

Collaborative Group for Studies on Yellow Fever Vaccine'; Luiz Antonio Bastos Camacho*.

${ }^{1}$ Colaboração multi-institucional;

${ }^{2}$ Fiocruz/ENSP.

Introduction: To assess the duration of immunity induced by yellow fever vaccine (YFV), the Brazilian Ministry of Health sponsored a 10-year follow-up study in areas without yellow fever circulation, where the YFV had not been implemented. Two other flavivirus (dengue and Zika viruses) have circulated in those areas and might interfere in the immunogenicity of YFV.

Objective: To ascertain the immune response 30-45 days after YFV and the influence of previous infections with dengue and Zika on YF antibody levels.

Methodology: In 2016-2018, we enrolled 2753 eligible children aged 9 to 59 months and 2009 adults aged 18 to 50 years in primary health care units at 3 municipalities in the state of Paraíba. The study staff followed current vaccination procedures recommended by the Ministry of Health. Laboratories at Fiocruz, Rio de Janeiro, performed serological tests for yellow fever (plaque reduction neutralization test), Zika and dengue (IgG, ELISA) before and 30-45 days after YFV injection.

Results: Before vaccination 1 child $(0.1 \%)$ and 26 adults (1.8\%) were seropositive to YF. After 30-45 days of vaccination, seropositive rates $(95 \%$ confidence limits) and geometric mean titers (GMT; reciprocal dilution) were $91.9 \%$ (89.8\%-93.6\%) and 511 (476-549) for 9-23-month-old children, 95.4\% (94.3\%$96.3 \%)$ and 689 (661-719) for 2-5-year-old children, 98.9\% (98.1\%-99.4\%) and 1162 (1126-1199) for 18-35-year-old adults and 99.3\% (98.4\%-99.8\%) and 1225 (1180-1272) for 36-50-year-old adults. In those age groups, $5.0 \%, 12.5 \%, 86.5 \%$ and $90.2 \%$, respectively, had dengue IgG before administration of YFV, whereas Zika IgG was detected in $0.9 \%, 18.8 \%, 65.3 \%$ and $69.5 \%$, respectively. YF seropositivity was $94.2 \%$ (90.7\%-96.6\%) and 94.4\% (93.4\%-95.3\%) in dengue IgG-positive and IgG-negative children, respectively. Corresponding GMT of YF antibodies were 631 (584-683) and 628 (602-654), respectively. Seropositive rates after YFV in adults were 98.9\% (98.3\%-99.3\%) in dengue seropositive and 100\% (98.2\%-100\%) in seronegative participants. YF antibody GMT (95\%C.I.) were 1188 (1158-1219) and 1169 (1082-1263) in dengue seropositive and seronegative adults, respectively. YF seropositivity was $94.2 \%(91.2 \%-96.4 \%)$ and $94.3 \%$ (93.2\%-95.2\%) for Zika-IgG-positive and negative children, respectively. Corresponding GMT were 647 (585-715) and 627 (603-652), respectively. Among Zika IgG-positive adults, seropositivity for YF was 98.8\% (98.0\%-99.3\%) whereas Zika IgG-negative adults were 99.5\% (98.6\%-99.9\%) YF-seropositive. YF antibody GMT (95\%C.I.) were 1161 (1124-1199) for Zika IgG-positive and 1234 (1191-1278) for IgG-negative adults. Adverse events were mostly mild, and eight severe cases had no plausible causal association with the vaccine.

Conclusion: YFV proved safe and immunogenic in a YF nonendemic area of Northeast Brazil, where a large proportion of the population had been infected by dengue and Zika. Previous infection with those Flaviviruses did not show meaningful impact on immune response to YFV. The lower seroconversion rates and GMT in children support recommendation of a booster dose of YFV.

Keywords: Yellow Fever Vaccine; Immune response; Flaviviruses 